\title{
Equation between Incisor Inclination Correction and Anteroposterior Movement of Point A to determine the Actual Magnitude of Maxillomandibular Difference
}

\author{
${ }^{1}$ Prashantha G Shivamurthy, ${ }^{2}$ Sathyashree Krishnamurthy, ${ }^{3}$ Pooja Mehta, ${ }^{4}$ Silju Mathew
}

\begin{abstract}
Aim: To determine the actual position of point $A$ by performing incisor inclination correction in class II division 2 and class III. Also, to determine the relation between the degree of incisor inclination correction and anteroposterior movement of point A by studying pre- and postalignment lateral cephalograms.
\end{abstract}

Materials and methods: The pre- and postalignment lateral cephalograms of 33 class II division 2 and 33 class III patients treated orthodontically were traced manually and analyzed. The linear anteroposterior measurements of point $A$ and center of rotation in relation to the vertical reference plane and angular measurements of upper incisor to maxillary plane were calculated.

Results: In class II division 2 category, the mean change in inclination from pre- to postalignment was $15.27^{\circ}$, mean change in position of center of rotation was $-1.29 \mathrm{~mm}$, and mean change in position of point $A$ from pre- to postalignment was $-2.67 \mathrm{~mm}$. In class III category, the mean change in inclination from pre- to postalignment was $-5.85^{\circ}$, mean change in position of center of rotation from pre- to postalignment was $1.94 \mathrm{~mm}$, and mean change in position of point $A$ from pre- to postalignment was $1.77 \mathrm{~mm}$.

Conclusion: The results of the study confirmed that for every $10^{\circ}$ proclination of the upper incisor in class II division 2 , point A moves $0.3 \mathrm{~mm}$ palatally and for every $10^{\circ}$ retroclination of the upper incisor in class III, point A moves $0.73 \mathrm{~mm}$ labially.

Clinical significance: In the clinical scenario of severely retroclined/proclined incisors, point A cannot depict the actual anterior limit of maxilla. Hence, when we use SNA to determine the anteroposterior position of maxilla and ANB to determine maxillomandibular difference, invariably we get altered values. Therefore, it is necessary to find an equation between the degree of incisor inclination correction and anteroposterior movement of point $A$.

Keywords: Center of rotation, Class III, Class II division 2, Inclination, Point A.

How to cite this article: Shivamurthy $P G$, Krishnamurthy $S$, Mehta P, Mathew S. Equation between Incisor Inclination Correction and Anteroposterior Movement of Point $A$ to determine the Actual Magnitude of Maxillomandibular Difference. World J Dent 2017;8(4):300-303.

\footnotetext{
${ }^{1-4}$ Department of Orthodontics, Faculty of Dental Sciences M.S. Ramaiah University of Applied Sciences, Bengaluru Karnataka, India

Corresponding Author: Prashantha G Shivamurthy, Department of Orthodontics, Faculty of Dental Sciences, M.S. Ramaiah University of Applied Sciences, Bengaluru, Karnataka, India Phone: +919886242624, e-mail: pacchi77@yahoo.com
}

Source of support: Nil

Conflict of interest: None

\section{INTRODUCTION}

Subspinale, also known as point $\mathrm{A}$, is the midline point whose position may be influenced by the head position. ${ }^{1}$ It is the deepest point on the premaxilla between anterior nasal spine and superior prosthion whose manual location may vary among tracers. Some authors also state that point A is a dentoalveolar landmark, which is influenced by growth as well as dentoalveolar remodeling during orthodontic treatment. ${ }^{2}$ Although it is not an ideal reference point because of the above-said disadvantages, point A is still widely considered as a valid anterior limit of maxilla. ${ }^{1}$ Hence, to determine whether maxilla is positioned anteriorly or posteriorly compared with the cranium, the angle SNA is still widely used. ${ }^{3}$

As the position of point $\mathrm{A}$ is directly proportional to the inclination of maxillary incisors, ${ }^{4,5}$ in class II division 2 malocclusion with retroclined incisors and in class III malocclusion with proclined incisors (dentoalveolar compensation to skeletal discrepancy), point A cannot depict the actual anterior limit of maxilla. This has been confirmed by many authors, such as Freeman, Hasund, Ulstein, and Gazilerli who mention that the position of points A and B was influenced by the axial inclination of the incisors. ${ }^{6}$ Especially, in class II division 2, because of retroclined incisors, point A will more be anteriorly positioned. Hence, when we use SNA to determine the anteroposterior position of maxilla and ANB to determine maxillomandibular difference, invariably we get the higher values of SNA and ANB angles. Therefore, it is difficult to understand the actual position of maxilla and actual magnitude of maxillomandibular difference. A similar problem is encountered even in severely proclined incisors where point $\mathrm{A}$ is moved posteriorly to give an exaggerated class III reading.

\section{AIMS}

With the above background, the present study has been undertaken to:

- Determine the actual position of point A by performing incisor inclination correction. 
- Determine the relation between the degree of incisor inclination correction and anteroposterior movement of point A by studying prealignment and postalignment lateral cephalograms of class II division 2 and in class III patients.

\section{MATERIALS AND METHODS}

The study consists of prealignment and postalignment lateral cephalograms of 33 class II division 2 and 33 class III patients treated orthodontically, which were collected retrospectively from the department record room. The inclusion criteria were all subjects having either class II division 2 or class III incisor relationship with no transverse discrepancies or no detectable lateral and sagittal shifts. Subjects with any congenital abnormality and facial trauma and medically compromised patients were excluded from the study. Any dentition with missing/ extracted teeth was also excluded.

Lateral cephalograms were taken in centric occlusion with lips relaxed and in natural head position with Frankfort plane parallel to the floor. Cephalograms were made at a distance of $150 \mathrm{~cm}$ (focus/object distance) using Planmeca PM 2002 CC Proline Pan/Ceph with a voltage of $70 \mathrm{KV}$ and current of $10 \mathrm{ma}$.

Both pre- and postalignment radiographs were taken using the same cephalostat. Radiographs were of sufficient quality to allow easy identification of relevant landmarks. The patients ranged in age from 16 to 31 years, with an average of 23.5 years. Ethical approval was obtained from the Institutional Ethical Review Board.

The standardized radiographs were traced manually and analyzed. The identification of landmarks was done; planes and long axis of the upper incisor were constructed. The linear measurements of point $A$ to the vertical reference plane (perpendicular to true horizontal line passing through nasion) and angular measurement (upper incisor to maxillary plane) were done for pre-and postalignment cephalograms.

The center of rotation of incisor in each case is determined by superimposing pretreatment and postalignment tracings on sella-nasion plane at nasion. The point at which pretreatment long axis of incisor overlays with postalignment long axis is considered as center of rotation and transferred to both tracings, and their position is measured from the vertical reference plane.

The changes in the position of point A horizontally, change in incisal inclination, and determination of center of rotation were obtained by finding the difference between pre- and postalignment tracings. Correction factor for magnification was calculated as and when required for individual cephalogram and was applied to all linear measurements.
A tooth's inclination can be changed in many ways depending on the position of center of rotation. If the center of rotation is located near the root apex, then the tooth changes its inclination by movement of crown (tipping) and vice versa, if the center of rotation is located near the incisal edge, then the tooth changes its inclination by movement of root (torque). If the center of rotation is located anywhere on the tooth's long axis, then it will be by controlled/uncontrolled tipping depending on how near/far is the center of rotation from the root apex.

If an incisor changes its inclination with center of rotation being at root apex, then root tip of that incisor is hardly changed, and Point A also should not change significantly. Here, there is a change in inclination without change in position of point A. Moreover, if incisor moves bodily, there should be a change in position of point A without change in inclination. So to avoid this bias, even horizontal movement of center of rotation is considered along with movement of point A (to vertical reference plane drawn perpendicular to true horizontal line passing through nasion).

\section{Method Error}

To assess the error of localizing the reference points and for the analysis procedure, 10 randomly selected radiographs were retraced and remeasured after a month to determine the intraexaminer error. The casual errors were assessed using Dahlberg's (1940) formula, and systematic errors were ascertained using paired t-test similar to the recommendation of Houston. ${ }^{7}$ The casual errors of the method (Dahlberg's formula) did not exceed $0.77^{\circ}$ or $0.56 \mathrm{~mm}$.

\section{RESULTS}

The study consisted of 33 class II division 2 and 33 class III samples with an average age of 23.5 years and mean duration between pretreatment and postalignment being 11 months (Tables 1 and 2).

In class II division 2 category, the mean pretreatment of upper incisor inclination compared with the SN plane was $103.12^{\circ}$ and mean postalignment inclination was $118.39^{\circ}$. The mean change in inclination from pretreatment to postalignment was $15.27^{\circ}$.

In class II division 2 category, the mean pretreatment center of rotation in relation to vertical reference plane perpendicular to true horizontal line passing through nasion was $-3.32 \mathrm{~mm}$, and postalignment was $-4.61 \mathrm{~mm}$. The mean change in position of center of rotation from pre- to postalignment was $-1.29 \mathrm{~mm}$.

In class II division 2 category, the mean pretreatment position of point A compared with vertical reference plane perpendicular to true horizontal line passing 


\begin{tabular}{|c|c|c|c|c|c|c|}
\hline Class & $n$ & Mean $\pm S D$ & Mean difference & SE of difference & $t$-value ${ }^{*}$ & $p$-value \\
\hline \multicolumn{7}{|l|}{ Class II division 2} \\
\hline Postpoint A (mm) & 33 & $-3.21 \pm 4.222$ & -2.67 & 0.745 & -3.579 & 0.001 \\
\hline Prepoint A (mm) & 33 & $-0.55 \pm 4.466$ & & & & \\
\hline Postcenter of rotation (mm) & 33 & $-4.61 \pm 5.045$ & -1.29 & 0.567 & -2.270 & 0.030 \\
\hline Precenter of rotation (mm) & 33 & $-3.32 \pm 4.899$ & & & & \\
\hline Post-U1 angle (degree) & 33 & $118.39 \pm 10.428$ & 15.27 & 1.972 & 7.747 & $<0.001$ \\
\hline Pre-U1 angle (degree) & 33 & $103.12 \pm 8.817$ & & & & \\
\hline \multicolumn{7}{|l|}{ Class III } \\
\hline Postpoint A (mm) & 33 & $-1.48 \pm 4.549$ & 1.94 & 0.556 & 3.491 & 0.001 \\
\hline Prepoint A (mm) & 33 & $-3.42 \pm 4.902$ & & & & \\
\hline Postcenter of rotation (mm) & 33 & $-2.80 \pm 5.697$ & 1.77 & 0.714 & 2.482 & 0.018 \\
\hline Precenter of rotation (mm) & 33 & $-4.58 \pm 5.564$ & & & & \\
\hline Post-U1 angle (degree) & 33 & $117.82 \pm 7.804$ & -5.85 & 0.954 & -6.133 & $<0.001$ \\
\hline Pre-U1 angle (degree) & 33 & $123.67 \pm 8.608$ & & & & \\
\hline
\end{tabular}

SD: Standard deviation

Table 2: Results for effect of maxillary incisor inclination changes on point A position

\begin{tabular}{|c|c|c|c|c|c|c|}
\hline & \multirow[b]{2}{*}{ Effect of U1 angle change } & \multirow{2}{*}{$\begin{array}{l}\text { Coefficient } \mathrm{mm} \text { per } \\
\text { degree of } U 1 \text { angle }\end{array}$} & \multirow[b]{2}{*}{ Standard error } & \multirow[b]{2}{*}{$p$-value } & \multicolumn{2}{|c|}{$95 \%$ confidence interval for $B$} \\
\hline & & & & & Lower limit & Upper limit \\
\hline Class II division 2 & Change in U1 angle & 0.030 & 0.058 & 0.609 & -0.089 & 0.149 \\
\hline Class III & Change in $\mathrm{U} 1$ angle & -0.073 & 0.101 & 0.477 & -0.278 & 0.133 \\
\hline
\end{tabular}

through nasion was $-0.55 \mathrm{~mm}$, and postalignment was $-3.21 \mathrm{~mm}$. The mean change in position of point $\mathrm{A}$ from pre- to postalignment was $-2.67 \mathrm{~mm}$.

In the class III category, the mean pretreatment upper incisor inclination compared with SN plane was $123.67^{\circ}$ and postalignment was $117.82^{\circ}$. The mean change in inclination from pretreatment to postalignment was $-5.85^{\circ}$.

In class III category, the mean pretreatment point A position compared with the vertical reference plane perpendicular to true horizontal line passing through nasion was -3.42 and postalignment position was -1.48 . The mean change in position of center of rotation from pre to postalignment was $1.94 \mathrm{~mm}$.

In class III category, the mean pretreatment position of center of rotation compared with vertical reference plane perpendicular to true horizontal line passing through Nasion was -4.58 , and postalignment was -2.80 . The mean change in position of point A from pre- to postalignment was $1.77 \mathrm{~mm}$.

The results of the study confirmed that for every $10^{\circ}$ change in the upper incisor inclination (proclination) in class II division 2, point A moves $0.3 \mathrm{~mm}$ palatally and for every $10^{\circ}$ change in the upper incisor inclination (retroclination) in class III, point A moves $0.73 \mathrm{~mm}$ labially.

\section{DISCUSSION}

Earlier studies confirmed the direct relation between position of point A and inclination of incisor teeth. ${ }^{4}$
Many others correlated the forward movement of point A in response to the treatment of cleft and class III, face mask therapy. ${ }^{8-10}$ Few studies also attempted to find the effect of torquing of incisors on position of point $A .4,6,11-13$

But only very few investigators tried to establish a direct association between the degrees of changes in the inclination of incisor to movement of point $\mathrm{A} .{ }^{2}$ With this background in this study, we made an attempt to determine the relation between the degree of incisor inclination correction and anteroposterior movement of point $\mathrm{A}$ by studying pre- and postalignment lateral cephalograms of class II division 2 and in class III patients. The samples were divided into two groups of class II division 2 and class III based on incisor relation as the type of incisor torque and the direction of movement of point A will be in the opposite direction for these two clinical conditions.

With this equation, the actual position of point A can be determined by doing incisor inclination correction based on Steiner's recommendations so that the real maxillomandibular discrepancy can be calculated.

Other similar studies used statistical methods either with paired t-test and univariable linear regression analysis $^{6}$ or generalized estimating equations ${ }^{2}$ (form of multiple regression analysis) to overcome the influence of growth on position of point A. Nevertheless, the use of a statistical model to eliminate this bias is not very clear. Hence, to eliminate the bias of effect of growth on position of point $\mathrm{A}$, only nongrowing patients were considered in this study. 
The inclination of incisor can be changed in many ways, by moving the roots and keeping the incisal edges stationary or vice versa. So, consideration of center of rotation is very crucial here.

Studies have shown that point A follows the apex of the upper incisors, however, by only as much as half., ${ }^{5,12}$ This is especially so in the case of palatal torque done in class II division 2. Another observed fact is that the center of rotation is located close to bracket irrespective of type of torque.

Most of the studies even observed a relation between linear movement of point A and SNA, i.e., that every millimeter of backward movement of point $\mathrm{A}$ results in nearly $1^{\circ}$ reduction in SNA..$^{5,14-17}$

\section{CONCLUSION}

This study demonstrates that for every $10^{\circ}$ change in incisor inclination (proclination) in class II division 2, point A moves $0.3 \mathrm{~mm}$ palatally and for every $10^{\circ}$ change in incisor inclination (retroclination) in class III, point A moves $0.73 \mathrm{~mm}$ labially. Accordingly, the corrected position of point $\mathrm{A}$ has to be taken into consideration to determine the actual anteroposterior relation of the jaw bases.

\section{CLINICAL SIGNIFICANCE}

In the clinical scenario of severely retroclined/proclined incisors, point A cannot depict the actual anterior limit of maxilla. Hence, when we use SNA to determine the anteroposterior position of maxilla and ANB to determine maxillomandibular difference, invariably we get altered values. Therefore, it is necessary to find an equation between the degree of incisor inclination correction and anteroposterior movement of point $\mathrm{A}$.

\section{REFERENCES}

1. Jacobson RL, Jacobson A. Point A revisited. Am J Orthod 1980 Jan;77(1):92-96.
2. Al-Abdwani R, Moles DR, Noar JH. Change of incisor inclination effects on points A and B. Angle Orthod 2009 May;79(3):462-467.

3. Steiner CC. Cephalometrics for you and me. Am J Orthod 1953 Oct;39(10):729-755.

4. van der Linden FP. A study of roentgenocephalometric bony landmarks. Am J Orthod 1971 Feb;59(2):111-125.

5. Cangialosi TJ, Meistrell ME Jr. A cephalometric evaluation of hard-and soft-tissue changes during the third stage of Begg treatment. Am J Orthod 1982 Feb;81(2):124-129.

6. Erverdi N. A cephalometric study of changes in point A under the influence of upper incisor inclinations. J Nihon Univ Sch Dent 1991 Sep;33(3):160-165.

7. Houston WJ. The analysis of errors in orthodontic measurements. Am J Orthod 1983 May;83(5):382-390.

8. Haskell BS, Farman AG. Exploitation of the residual premaxillary-maxillary suture site in maxillary protraction. An hypothesis. Angle Orthod 1985 Apr;55(2):108-119.

9. Ricketts RM. Cephalometric synthesis: an exercise in stating objectives and planning treatment with tracings of head roentgenogram. Am J Orthod 1960 Sep;46(5):647-673.

10. Subtelny JD. Oral respiration: facial mal-development and corrective dento-facial orthopaedics. Angle Orthod 1980 Jul;50(3):147-164.

11. Chen Q, Zhang C, Zhou Y. The effects of incisor inclination changes on the position of point $\mathrm{A}$ in Class II division 2 malocclusion using three-dimensional evaluation: a longterm prospective study. Int J Clin Exp Med 2014 Oct;7(10): 3454-3460.

12. Bicakci AA, Cankaya OS, Mertoglu S, Yilmaz N, Altan BK. Does proclination of maxillary incisors really affect the sagittal position of Point A? Angle Orthod 2013 Nov;83(6):943-947.

13. Al-Nimri KS, Hazza'a AM, Al-Omari RM. Maxillary incisor proclination effect on the position of point A in Class II division 2 malocclusion. Angle Orthod 2009 Sep;79(5):880-884.

14. Mitchell DL, Kinder JD. A comparison of two torquing techniques on the maxillary central incisor. Am J Orthod 1973 Apr;63(4):407-413.

15. Weber FN. Clinical investigations related to use of the Begg technique at the university of Tennessee. Am J Orthod 1971 Jan;59(1):24-36.

16. Barton JJ. A cephalometric comparison of cases treated with edgewise and Begg technique. Angle Orthod 1973 Jan;43(1):119-126.

17. Venezia AJ. Pure begg and edgewise arch treatments: comparison of results. Angle Orthod 1973 Jul;43(3):289-300. 